\title{
Sun shadow positioning technology
}

\author{
Ben Niu ${ }^{1, a}$ \\ ${ }^{1}$ School of North China Electric Power University, Baoding 071000, China \\ a2676190951@qq.com
}

Keywords: Solar elevation angle Azimuth Simulated annealing algorithm Image Processing

\begin{abstract}
We set up a mathematical model in connection with sun shadow positioning technology and apply it to solving analytic function and parallel simulated annealing algorithm to establish the optimization Model with the objective function of the angles produced by the shadow changes and the minimum error between the actual data and the change of shadow length ratio according to the data in 2015 National Mathematical Contest in Modeling title A.
\end{abstract}

\section{Introduction}

As digital image were used more and more widely in people's lives, it is an important aspect of video data analysis that how to determine the location and the date of shooting video. The sun shadow sun shadow positioning technology is a method to determine the location and the date of video shooting through analysis of the changes of objects' sun shadow in the video. In this paper, mathematical formulas and model algorithms are used to find the shooting location of the image by the partially known variables. The confirmation of accurate real-time shooting position is of important practical significance and great value for both technology and human social life.

\section{Solving of solar elevation angle and azimuth}

For a place on Earth, the solar elevation angle refers to the angle between incidence direction of sunlight and the ground-piston; that is the angle [1] between the sun's rays of a place and the surface tangent connected with the earth's core through that place.

Solar azimuth, the orientation of the location of sun, is defined as the angle between sun's rays projected on the ground-piston and the local meridian; it can be approximately regarded as the angle between the shadows of a straight line erected on the ground and due south. The time difference $\mathrm{E}_{\mathrm{Q}}$ [2] refers to the difference between the apparent solar time and local mean solar time. The formula is as follows:

$$
\left\{\begin{array}{l}
E_{Q}=0.0028-1.9857 \sin Q+9.9059 \sin 2 Q-7.0924 \cos Q-0.6882 \cos 2 Q \\
Q=2 \pi * 57.3\left(N+\Delta N-N_{0}\right) / 365.2422
\end{array}\right.
$$

$\mathrm{N}$ in the formula refers to the number of days arranged in order, 0 for $1^{\text {st }}$ January, 1 for $2^{\text {nd }}$ January while others are by analogy, 364(non leap year) or 365(leap year) for 31th, December.

$\Delta N$, integrated day, consists of a corrected value ' $L$ ' of time difference set by the difference between the observation point and the Greenwich longitude and a ' $\mathrm{W}$ ' resulted from the difference between the observation time and Greenwich time of zero o'clock.

$$
\pm L=(D+M / 60) / 15
$$

$\mathrm{D}$ in the formula refers to the difference of the observation points' longitude. $\mathrm{M}$ refers to minutes and can be converted into the time difference of Greenwich $\mathrm{L}_{0}$. Minus stands for east longitude while positive stands for west.

$W=S+F / 60$. S refers to the hour time of observation while $\mathrm{F}$ stands for the minute. The last two hour times are combined into the point of the day. China is in the east longitude so $\mathrm{L}$ is negative. Therefore:

$$
\left\{\begin{array}{l}
\Delta N=(W-L) / 24 \\
N_{0}=79.6764+0.2422(Y-1985)-I N T[0.25-(Y-1985)]
\end{array}\right.
$$

$\mathrm{Y}$ refers to the year. $\mathrm{INT}(\mathrm{X})$ refers to the maximum integer which is not greater than $\mathrm{X}$. Apparent solar time[3] 


$$
T T=T_{w}+E_{Q}=C_{T}+L_{C}+E_{Q}
$$

$T_{W}$ refers to local mean solar time while $C_{T}$ refers to local standard time. China regarded the time of $120^{\circ} \mathrm{E}$ as Chinese standard time. There are some time differences between the local time of Beijing (116 $\left.{ }^{\circ} 3^{\prime} 29^{\prime \prime}\right)$ and Chinese standard time. $\mathrm{L}_{C}$ refers to the longitude revised (4min/degree). If the local meridian circle is in the east of standard meridian circle, LC will be positive. If not, LC will be negative.

EQ refers to the time difference.

Declination $\mathrm{D}_{\mathrm{E}}$,

$$
\begin{aligned}
D_{E}= & 0.3723+23.2567 \sin Q+0.1149 \sin 2 Q-0.1712 \sin 3 Q-0.7580 \cos Q \\
& +0.3656 \cos 2 Q+0.0201 \cos 3 Q
\end{aligned}
$$

$\mathrm{Q}$ is the same as the $\mathrm{Q}$ in the (1)

The formula for solar elevation angle $\mathrm{H}_{\mathrm{A}}$ and azimuth $\mathrm{A}$ are:

$$
\left\{\begin{array}{l}
\sin H_{A}=\sin \Phi \bullet \sin D_{E}+\cos \Phi \bullet \cos D_{E} \bullet \cos T_{0} \\
\cos A=\left(\sin H_{A} \bullet \sin \Phi-\sin D_{E}\right) / \cos H_{A} \cos \Phi
\end{array}\right.
$$

$\Phi$ refers to latitude; DE refers to solar declination; T0 refers to solar hour angle. $T_{0}=(T T-12) \times 15^{0}$.

\section{Solve shadow's length and direction}

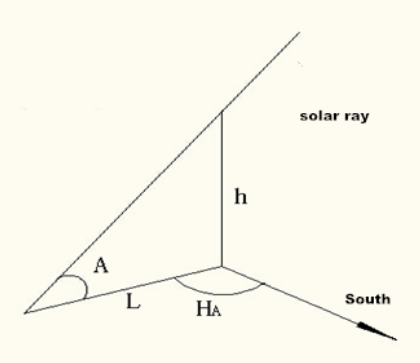

Figure 1. Shadow sketch map

In the figure above, straight rod's height is h, and shadow's length is L, and the sun's rays angle with the ground is A, so the length of shadow $L=\frac{h}{\tan A} \quad$,The Angle of shadow and due south is $H_{A}$.h is known, we can calculate shadow's length and position by calculating A and $H_{A}$.

\section{Search based on improved simulated annealing algorithm}

\subsection{The establishment of objective function.}

We do not consider the pointing directions of $\mathrm{X}$ and $\mathrm{Y}$ and establish a rectangular coordinate system. In the different direction of the coordinate system, the angle between shadows and the change ratio of shadow's length which can be calculated from coordinates are the same.

The given date is April 18, 2015. Declination DE can be calculated from formula (1). Given any latitude and longitude according, the corresponding local solar azimuth for the moment can be calculated from formula (1) (2) (3) (4) (6). The difference value of azimuth angles of different time is the angle between shadows. Similarly, the ratio of elevating angles of different time is the ratio of the shadows' length. The closer the calculated results and results from the given data are, the smaller the error is.

The data give 21 times. For any given coordinate of latitude and longitude, the ith time, the shadow angle $\theta \mathrm{i}$ of the next time and the shadow length ratio $\lambda \mathrm{i}$ can be found. According to the given data, the ith time, the shadow angle $\theta \mathrm{i} 0$ of the next time and the shadow length ratio $\lambda \mathrm{i} 0$ can be determined.

Finally, the objective function is: 


$$
\min \sum_{i=1}^{20}\left[\left(\theta_{i}-\theta_{i 0}\right)^{2}+\left(\lambda_{i}-\lambda_{i 0}\right)^{2}\right]
$$

\subsection{The setting of searching coordinate}

In the searching range determined in the preparation of model, give the latitude and longitude coordinates of all the points to be calculated. Latitude and longitude are divided by 0.01 degree step length. For instance, the given latitude and longitude coordinate is $\left(110.03^{0} \mathrm{E}, 15.24^{0} \mathrm{~S}\right)$ or $\left(90.53^{0} \mathrm{E}, 45.25^{0} \mathrm{~N}\right)$.

Meanwhile, we could look up the map and calculate the latitude and longitude range of daytime hemisphere in the given time to define the initial constraint condition.

Longitude: $10^{\circ}$ east longitude to $130^{\circ}$ east longitude east longitude.

\section{The results of the model and analysis}

\subsection{The results of the model}

Set $\mathrm{N}=24$, use the parallel simulated annealing algorithm and get a disaggregation. The expectation of disaggregation is $\left(109.4^{\circ} \mathrm{E}, 18.08^{\circ} \mathrm{N}\right)$,that is the possible place.

\subsection{Analysis of the result}

When using the parallel simulated annealing algorithm, although the final results will have deviation if the initial value is different, the results will be within a certain range, it meets the requirement of accuracy.

The curve fitting of optimal solutions has been made, the figure is as follows:
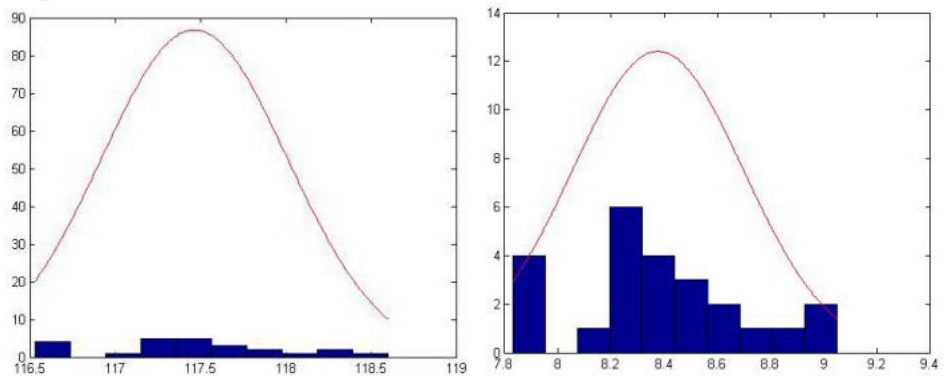

Figure2.Longitude and latitude’s fitting

It can be seen from figure 2 that the longitude and latitude of the 24 coordinates obey the normal distribution, which in accordance with the expected result. The expected values can be regarded as the optimal solution.

Regard expectation $\left(109.4^{\circ} \mathrm{E}, 18.08{ }^{\circ} \mathrm{N}\right)$ as a known condition and take the latitude and longitude coordinates back to the model, the straight rod sun's shadow length change curve of Beijing time 14:42-15:42 can be drawn out. At the same time, draw the sun shadow length change curve based on the shadow vertex coordinates given in the appendix 1 in the same figure, compare the two curves.

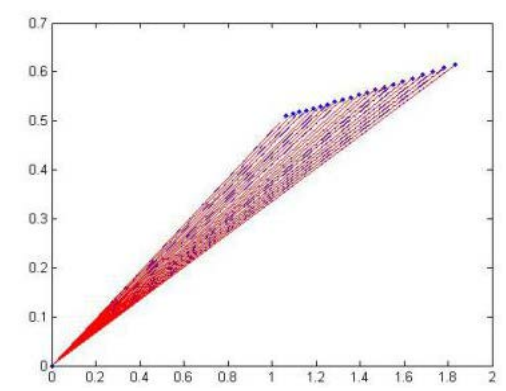

Figure3.The shadow length change curve comparison chart 


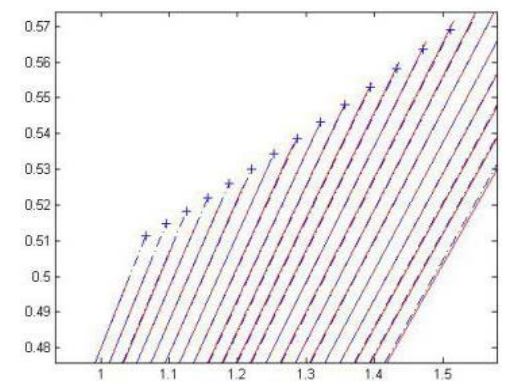

Figure4. Contrast figure of local amplification

Form figure 3 and figure 4, we can see that the straight rod sun's shadow length change curve which is found by regarding the expectation as known condition and the sun shadow length change curve based on the shadow vertex coordinates given in the appendix 1 are highly accordant. It declares that the expectation highly conforms to possible location coordinates in the issue, the several possible latitude and longitude coordinates conform to the subject's requirements.

From the analysis above, parallel simulated annealing algorithm can avoid accidental error and the its results are highly reliable.

\section{References}

[1] Chuang Zhang. The sun position calculation and real-time application in the image illumination direction _ Chuang Zhang [J]. Journal of photoelectric measurement technology, 2010, 33 (11) : 87-88

[2] The China meteorological administration. The ground meteorological observation specification [M]. Beijing: meteorological press, 2003.

[3] True solar time, http://www.baidu.com/s? Wd = true solar time \& ie = utf-8

[4] Shoukui Si, Xiqing Sun. Mathematical modeling algorithm and application. Beijing: national defence industry press, 2015.3 reprint

[5] Feng Pang. The principle of simulated annealing algorithm and the application of the algorithm in the optimization problem _ Feng Pang [J]. J, 2005:8-9. 